\title{
Effect of postoperative goal-directed therapy in cancer patients undergoing high-risk surgery: a randomized clinical trial and meta-analysis
}

Aline Rejane Muller Gerent ${ }^{1}$, Juliano Pinheiro Almeida', Evgeny Fominskiy ${ }^{2}$, Giovanni Landoni ${ }^{2}$ Gisele Queiroz de Oliveira', Stephanie Itala Rizk', Julia Tizue Fukushima', Claudia Marques Simoes', Ulysses Ribeiro $\mathrm{Jr}^{3}$, Clarice Lee Park', Rosana Ely Nakamura', Rafael Alves Franco ${ }^{1}$, Patricia Inês Cândido', Cintia Rosa Tavares ${ }^{1}$, Ligia Camara', Graziela dos Santos Rocha Ferreira', Elisangela Pinto Marinho de Almeida', Roberto Kalil Filho ${ }^{4}$, Filomena Regina Barbosa Gomes Galas ${ }^{1}$ and Ludhmila Abrahão Hajjar ${ }^{4^{*}}$

\begin{abstract}
Background: Perioperative goal-directed hemodynamic therapy (GDHT) has been advocated in high-risk patients undergoing noncardiac surgery to reduce postoperative morbidity and mortality. We hypothesized that using cardiac index (Cl)-guided GDHT in the postoperative period for patients undergoing high-risk surgery for cancer treatment would reduce 30-day mortality and postoperative complications.

Methods: A randomized, parallel-group, superiority trial was performed in a tertiary oncology hospital. All adult patients undergoing high-risk cancer surgery who required intensive care unit admission were randomly allocated to a Cl-guided GDHT group or to a usual care group. In the GDHT group, postoperative therapy aimed at $\mathrm{Cl} \geq 2.5$ $\mathrm{L} / \mathrm{min} / \mathrm{m}^{2}$ using fluids, inotropes and red blood cells during the first 8 postoperative hours. The primary outcome was a composite endpoint of 30-day all-cause mortality and severe postoperative complications during the hospital stay. A meta-analysis was also conducted including all randomized trials of postoperative GDHT published from 1966 to May 2017.

Results: A total of 128 patients (64 in each group) were randomized. The primary outcome occurred in 34 patients of the GDHT group and in 28 patients of the usual care group (53.1\% vs $43.8 \%$, absolute difference $9.4(95 \% \mathrm{Cl},-7.8$ to 25.8); $p=0.3$ ). During the 8 -h intervention period more patients in the GDHT group received dobutamine when compared to the usual care group (55\% vs $16 \%, p<0.001$ ). A meta-analysis of nine randomized trials showed no differences in postoperative mortality (risk ratio $0.85,95 \% \mathrm{Cl} 0.59-1.23 ; p=0.4 ; p$ for heterogeneity $=0.7 ; P^{2}=0 \%$ ) and in the overall complications rate (risk ratio $0.88,95 \% \mathrm{Cl} 0.71-1.08 ; p=0.2 ; p$ for heterogeneity $=0.07 ; P^{2}=48 \%$ ), but a reduced hospital length of stay in the GDHT group (mean difference (MD) $-1.6 ; 95 \% \mathrm{Cl}-2.75$ to $-0.46 ; p=0.006 ; p$ for heterogeneity $=0.002 ; l^{2}=74 \%$ ).

(Continued on next page)
\end{abstract}

\footnotetext{
* Correspondence: ludhmila@terra.com.br

${ }^{4}$ Department of Cardiopneumology, Instituto do Coracao, Hospital das

Clinicas, Faculdade de Medicina da Universidade de Sao Paulo, Sao Paulo,

Brazil

Full list of author information is available at the end of the article
}

(C) The Author(s). 2018 Open Access This article is distributed under the terms of the Creative Commons Attribution 4.0 International License (http://creativecommons.org/licenses/by/4.0/), which permits unrestricted use, distribution, and reproduction in any medium, provided you give appropriate credit to the original author(s) and the source, provide a link to the Creative Commons license, and indicate if changes were made. The Creative Commons Public Domain Dedication waiver (http://creativecommons.org/publicdomain/zero/1.0/) applies to the data made available in this article, unless otherwise stated. 
(Continued from previous page)

Conclusions: Cl-guided hemodynamic therapy in the first 8 postoperative hours does not reduce 30-day mortality and severe complications during hospital stay when compared to usual care in cancer patients undergoing high-risk surgery.

Trial registration: www.clinicaltrials.gov, NCT01946269. Registered on 16 September 2013.

Keywords: Goal-directed therapy, Cancer, High-risk surgery, Mortality, Randomized clinical trial, Meta-analysis

\section{Background}

For many patients with solid tumors, surgery remains the mainstay of therapy. For these patients, a complication-free operative procedure is vital to maximize the chances that oncological treatment is successful. Postoperative care of cancer patients having major abdominal surgery is challenging because of the unusually long duration of the surgical procedures, the significant fluid and blood losses that can occur, the inherent immunological disturbs and the increased operative risk, as demonstrated by previous studies $[1,2]$.

Goal-directed hemodynamic therapy (GDHT) comprising rational use of fluids, inotropes, vasopressors and red blood cell (RBC) transfusion according to hemodynamic targets to improve oxygen delivery $\left(\mathrm{DO}_{2}\right)$ can provide outcome benefits in high-risk patients [3]. Even if some studies suggested that GDHT in high-risk patients undergoing surgery was associated with a significant reduction in morbidity and mortality [4-6], recent evidence suggests that the benefits are less than previously hypothesized because of the potential harm of fluid overload, drugs side effects and invasive monitoring $[7,8]$.

In addition, in most studies GDHT was investigated during surgery and extending for the first 6-12 $\mathrm{h}$ after intensive care unit (ICU) admission. It is unclear whether the potential benefits of GDHT are present when GDHT is used during the whole perioperative period or if GDHT use only in the postoperative ICU setting might result in clinical benefits or even in harmful interventions.

Therefore, we performed a randomized clinical trial (RCT) to evaluate the effects of a postoperative GDHT protocol on a composite endpoint of 30-day all-cause mortality and severe postoperative complications during the hospital stay in high-risk cancer patients undergoing surgery. We also conducted an updated systematic review focusing on postoperative GDHT and incorporating the findings of this trial.

\section{Methods}

The Goal-Directed Resuscitation in Cancer Surgery (GRICS II) trial was a single-center, parallel and randomized trial performed at the Cancer Institute, University of Sao Paulo, Sao Paulo, Brazil. The study was approved by the Faculty of Medicine Ethics Committee (number 335/13). This trial was registered at ClinicalTrials.gov on September 16, 2013 (NCT01946269). Written informed consent was obtained from all subjects or their legal surrogates prior to enrolment in the study. Patients were enrolled from October 2013 to September 2015. The trial was overseen by an independent data and safety monitoring board. The two funding sources were the University of Sao Paulo (Brazil) and Edwards LTDA (Irvine, CA, USA), which had no other role in the study. All the authors vouch for the fidelity of the study to the trial protocol and for the accuracy of the data and data analyses.

\section{Participants}

Consecutive patients who were 18 years old or more and scheduled for cancer surgery at the Cancer Institute were screened for enrolment. Patients undergoing high-risk surgery for cancer treatment (upper and lower gastrointestinal tract, urogenital tract, liver or biliary tract surgeries) with duration longer than $90 \mathrm{~min}$ and requiring ICU admission were included in the study. Patients were excluded from the study if one of the following was present: declined consent; emergency surgery; enrolled in another study; weight under $55 \mathrm{~kg}$ or over $140 \mathrm{~kg}$; active bleeding; contraindication to invasive hemodynamic monitoring; hemodynamic instability (norepinephrine higher than $1 \mu \mathrm{g} / \mathrm{kg} / \mathrm{min}$ ); surgery for palliative treatment only; or presence of cardiac arrhythmia (atrial fibrillation, frequent ectopy or other dysrhythmias).

\section{Randomization}

Randomization was performed, with a computer-generated list in a 1:1 ratio generated online by a web-based program that ensured allocation concealment.

The investigator opened the serially numbered, sealed, opaque envelopes on arrival of the patients at the ICU, provided exclusion criteria were not met. The nature of the intervention precluded blinding of the patients and attending physicians. Outcome assessors were unaware of the assigned treatment.

The decision to admit the patients to the ICU was made by clinical staff and registered before randomization and surgery.

\section{Study treatments All patients}

Anesthetic management and perioperative care of patients were standardized and are described in detail in 
Additional file 1: Appendix 1. Briefly, all patients were monitored with a central venous line and indwelling radial artery catheter. Fluids, vasopressor and inotropic agents were administered to maintain mean arterial pressure $(\mathrm{MAP}) \geq 65 \mathrm{mmHg}$, urinary output $>0.5 \mathrm{ml} / \mathrm{kg} / \mathrm{h}$, oxygen venous saturation $\geq 70 \%$ and lactate levels $<3$ $\mathrm{mmol} / \mathrm{L}$. Preload was optimized by fluid loading until pressure pulse variation (PPV) was $<10 \%$. Interventions included fluid resuscitation with lactated Ringer's preferentially, administration of dobutamine and RBC transfusion if needed according to hemodynamic data.

After ICU admission, all patients received care to keep adequate temperature $\left(36{ }^{\circ} \mathrm{C}\right)$, oxygen saturation $\geq 95 \%$, MAP $\geq 65 \mathrm{mmHg}$, heart rate (HR) between 70 and $100 \mathrm{bpm}$, central venous oxygen saturation $\left(\mathrm{ScvO}_{2}\right)>70 \%$, lactate level $<3 \mathrm{mmol} / \mathrm{L}$, urinary output $>0.5 \mathrm{ml} / \mathrm{kg} / \mathrm{h}$ and normoglycemia (140-180 mmol/L). Additional fluids and vasoactive drugs could be administered by the physicians guided by HR, MAP, urine output, venous central oxygen saturation, serum lactate and base excess. Analgesia was provided in most cases by epidural infusion. In a few cases when an epidural technique was not possible, intravenous infusion of morphine was performed.

\section{GHDT group}

A cardiac output monitor - Vigileo - Edwards LTDA (Irvine, CA, USA) was connected to the radial artery and the cardiac index $(\mathrm{CI})$ and stroke volume index (SVI) values were used as goals to deliver the hemodynamic intervention.

The protocol started at ICU admission and lasted $8 \mathrm{~h}$. The main goal was to achieve $\mathrm{CI} \geq 2.5 \mathrm{~L} / \mathrm{min} / \mathrm{m}^{2}$. Initially, fluid resuscitation was provided with the administration of an intravenous bolus in $15 \mathrm{~min}$ of $200 \mathrm{ml}$ of Ringer lactate solution with $50 \mathrm{ml}$ of $20 \%$ albumin solution if the SVI was less than $35 \mathrm{ml} / \mathrm{m}^{2}$. Fluid administration was repeated accordingly to responsiveness and interrupted if SVI $\geq 35 \mathrm{ml} / \mathrm{m}^{2}$. The definition of positive response to fluid challenge was an increase in cardiac index $\geq 15 \%$ from baseline.

If the $\mathrm{CI}$ remained $<2.5 \mathrm{~L} / \mathrm{min} / \mathrm{m}^{2}$, dobutamine infusion was started at $3 \mu \mathrm{g} / \mathrm{kg} / \mathrm{min}$ and titrated every $15 \mathrm{~min}$ up to a maximum dose of $20 \mu \mathrm{g} / \mathrm{kg} / \mathrm{min}$. If these interventions did not result in $\mathrm{CI} \geq 2.5 \mathrm{~L} / \mathrm{min} / \mathrm{m}^{2}$, RBCs were transfused 1 unit at a time to achieve hematocrit > 24\% (Fig. 1).

\section{Usual care group}

For patients in the usual care group, the institutional protocol was followed to achieve HR between 70 and $100 \mathrm{bpm}, \quad \mathrm{ScvO}_{2}>70 \%$, lactate level $<3 \mathrm{mmol} / \mathrm{L}$, hematocrit value $>28 \%, \mathrm{MAP} \geq 65 \mathrm{mmHg}$ and urinary output $>0.5 \mathrm{ml} / \mathrm{kg} / \mathrm{h}$ through fluid resuscitation with
Ringer lactate solution, administration of dobutamine and $\mathrm{RBC}$ transfusion.

\section{Study outcomes}

The primary endpoint was a composite endpoint of 30-day mortality and major clinical complications (Additional file 1: Appendix 2) during the hospital stay. Clinical complications were acute kidney injury (AKI), stroke, myocardial infarction, acute decompensated heart failure, pulmonary thromboembolism, mesenteric ischemia, peripheral vascular ischemia, acute respiratory distress syndrome (ARDS), deep wound infection and reoperation.

Secondary endpoints included the incidence of septic shock, development of AKI requiring renal replacement therapy (RRT), ICU readmission rate, ICU and hospital lengths of stay (LOS), 7-day sepsis-related organ failure assessment (SOFA) score and 90-day mortality (Additional file 1: Appendix 2).

We also analyzed the following hemodynamic parameters and perfusion indexes during the intervention period (at $0 \mathrm{~h}, 2 \mathrm{~h}$, and $8 \mathrm{~h}$ ): $\mathrm{HR}, \mathrm{MAP}, \mathrm{ScvO}_{2}$, lactate levels, base excess and $\mathrm{CO}_{2}$ gap.

\section{Data collection and definition of complications}

Data were collected by two blinded assessors with more than 3 years of work experience in the cancer ICU. Patients were discharged from the ICU if their physiologic status was stable, there was no need for monitoring and active interventions were not planned. Follow-up after hospital discharge was performed by telephone on the 30th postoperative day (Additional file 1: Appendix 3).

\section{Systematic review}

We also conducted a meta-analysis of all published RCTs following the PRISMA guidelines and comparing postoperative GDHT with standard therapy in noncardiac surgery adult high-risk patients. The PubMed/Medline, Embase and Scopus databases were searched from inception up to May 1, 2017. Details on the PubMed search strategy are provided in Additional file 1: Appendix 4. Two researchers independently screened the titles and abstracts of all initially identified studies according to the selection criteria and extracted the required data. Consensus was reached in the case of any inconsistency with involvement of a third author. We included only studies reporting mortality data (primary outcome of the meta-analysis). Secondary endpoints were overall complications rate and hospital length of stay. Detailed methods are described in Additional file 1: Appendix 4.

\section{Statistical analysis}

Considering a two-sided alpha level of 0.05 and a statistical power of $90 \%$, we calculated that a sample size of 


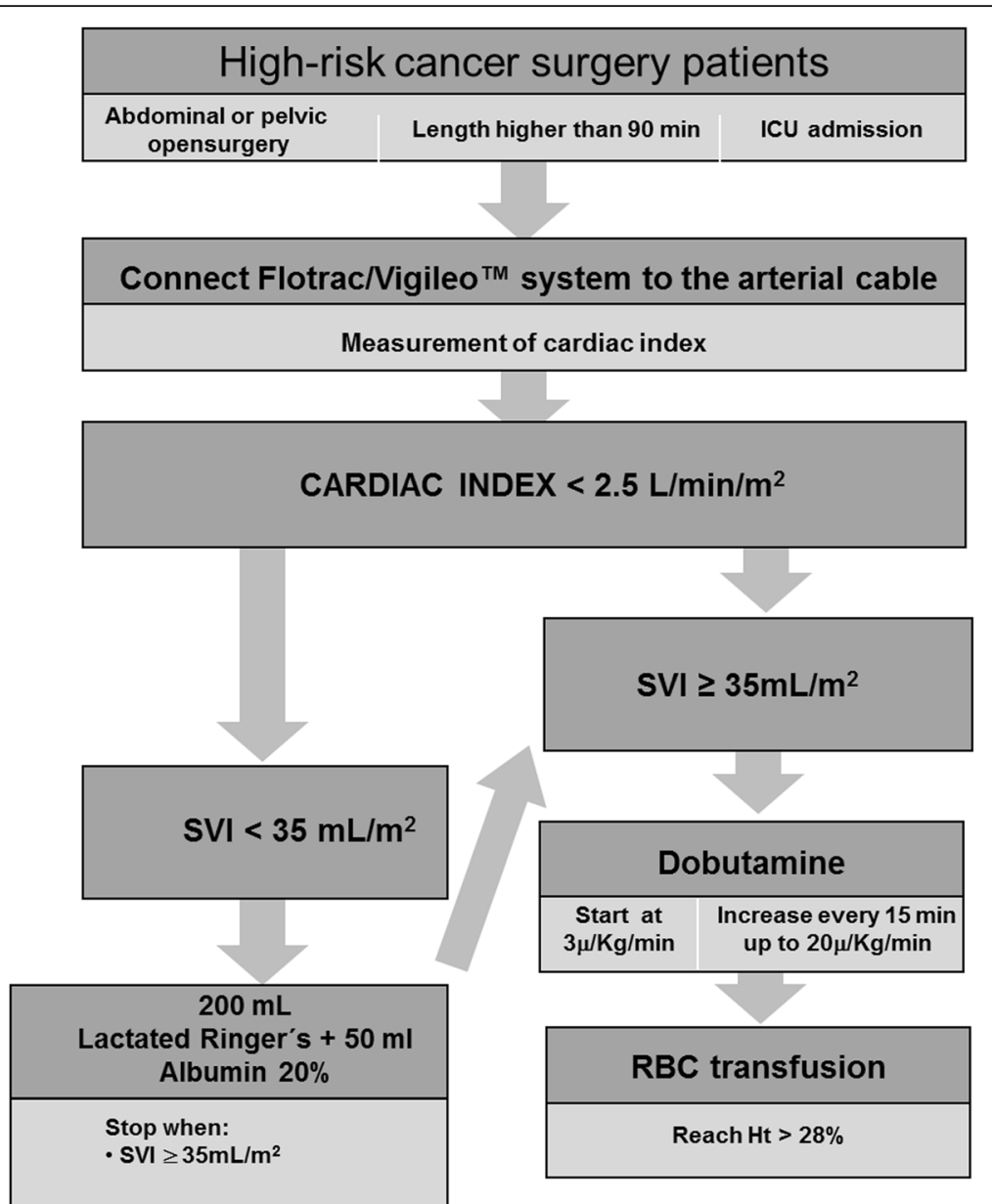

Fig. 1 Algorithm of treatment in the goal-directed hemodynamic therapy (GDHT) group. ICU intensive care unit, SVI stroke volume index, RBC red blood cell, Ht hematocrit

128 patients would be required to reduce the incidence of the composite endpoint from $56 \%$ in the control group to $28 \%$ in the GDHT group [5]. All analyses were conducted according to the intention-to-treat principle. No assumptions were made for missing or unavailable data. We report continuous variables as mean with standard deviation (SD) or median with interquartile range (IQR), and categorical variables as proportions. Continuous variables were compared using a Student's $t$ test or Mann-Whitney $U$ test and categorical variables using Pearson $X^{2}$ or Fisher exact or likelihood ratio test. Comparisons of SOFA score over time were made using nonparametric Friedman test. Kaplan-Meier curves were built for event-free survival probability up to 30 days following surgery. Two-sided $p<0.05$ was considered statistically significant.

For the meta-analysis, treatment effects were reported as risk ratio (RR) with $95 \%$ confidence interval (CI) for mortality and complications or mean differences with standard deviations for hospital length of stay. The Mantel-Haenszel method was used to combine summary measures using a random-effects model. We evaluated heterogeneity between studies using Cochran's $Q$ and the $I^{2}$ statistic. We assessed the potential of publication bias through funnel plot generation and Egger's regression test.

SPSS version 18.0 (SPSS Inc., Chicago, IL, USA), Review Manager (RevMan, Version 5.3, 2014; The Nordic Cochrane Centre, The Cochrane Collaboration, Copenhagen, Denmark) and STATA (Version 11; StataCorp, College Station, TX, USA) were used for all statistical analyses.

\section{Results}

\section{Study population}

One hundred and twenty-eight patients were included in the study (Fig. 2). All patients had outcome data collected and completed the follow-up. Patients were 67 years old and had a good performance status prior to surgery (Table 1), the most commonly performed being gastrointestinal followed by liver and biliary tract surgery. The groups were similar regarding the 


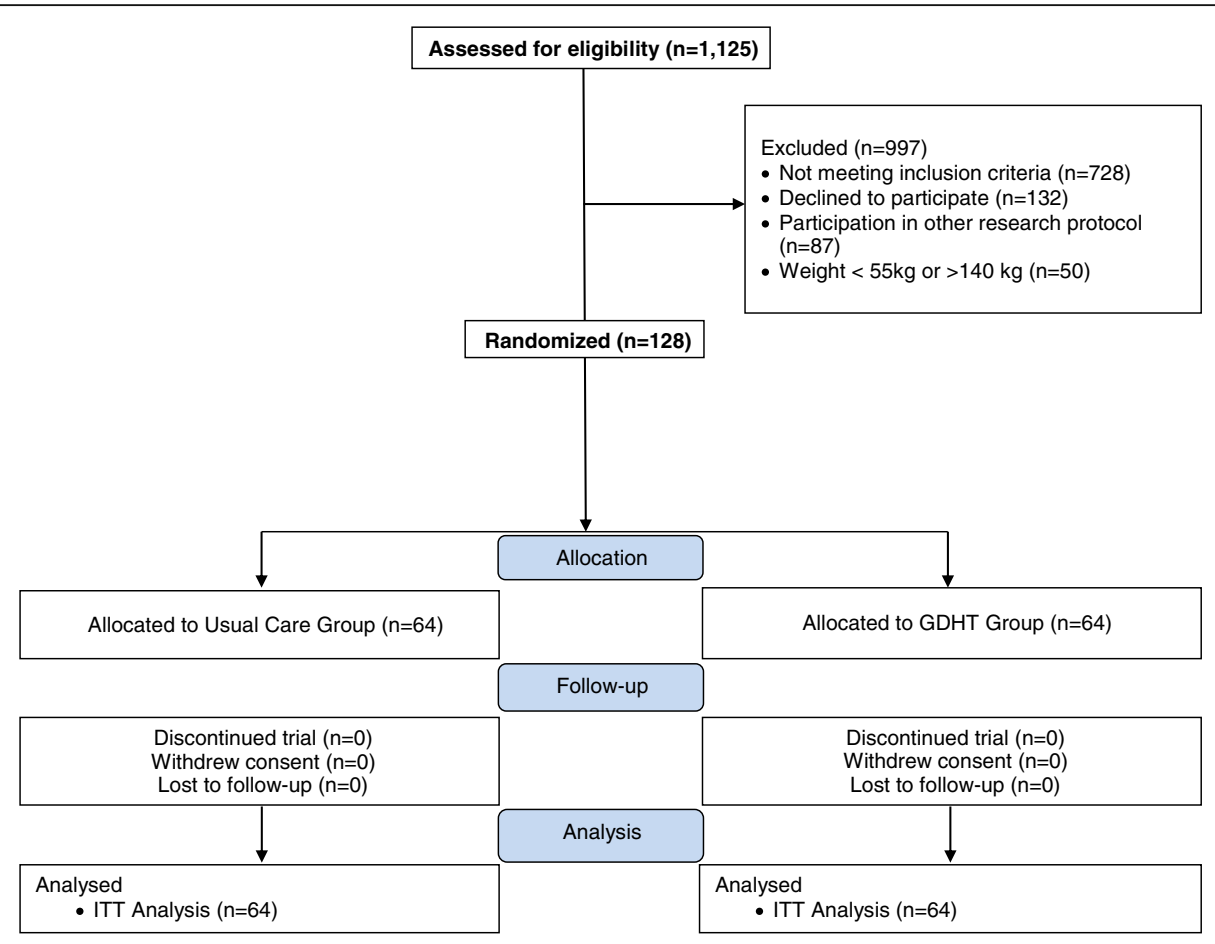

Fig. 2 Study flow chart. GDHT goal-directed hemodynamic therapy, ITT intention-to-treat

intraoperative amount of fluids, vasoactive drugs and RBC transfusion received (Table 2).

\section{Intervention period}

In the first $8 \mathrm{~h}$ after ICU admission, there was no difference between groups in the volume of administered fluids $(1195 \pm 719 \mathrm{ml}$ GDHT group vs $1290 \pm 609 \mathrm{ml}$ usual care group, $p=0.4$ ) and in the RBC transfusion rate (3.1\% GDHT group vs $0 \%$ usual care group, $p=0.5$ ). There was a higher use of dobutamine in the GDHT group when compared to the usual care group (35 patients $(55 \%)$ vs 10 patients $(15 \%), p<0.001)$. The CI of the GDHT group patients during the 8 -h intervention is reported in Additional file 1: Figure S1. There were no differences between groups in the hemodynamic parameters or perfusion indexes (HR, MAP, $\mathrm{ScvO}_{2}$, lactate levels, base excess and $\mathrm{PCO}_{2}$ gap; Table 3).

\section{Primary outcome}

The primary composite endpoint of 30-day all-cause mortality and severe clinical complications occurred in 34 patients $(53 \%)$ in the GDHT group and in 28 patients $(44 \%)$ in the usual care group $(p=0.3)$. No significant differences were observed between the GDHT and usual care groups in the rate of mortality ( $14 \%$ vs $9 \%, p=0.4)$ and of the other severe complications (Table 4), with AKI $(51 \%$ vs $39 \%, p=0.184)$ and reoperation ( $8 \%$ vs $5 \%$, $p=0.7$ ) being the most frequently represented.

\section{Secondary outcomes}

We did not observe significant differences in any of the secondary outcomes between the GDHT and usual care groups: septic shock ( $16 \%$ vs $13 \%, p=0.6)$, AKI needing RRT (13\% vs $8 \%, p=0.4)$, ICU readmission ( $13 \%$ vs $9 \%$, $p=0.6)$, ICU stay $(3(2-8)$ days vs $3(2-5)$ days, $p=0.6)$ and hospital LOS (11 (6-19) days vs 10 (6-15) days, $p=0.4$; Table 5).

\section{Meta-analysis}

Our searches identified 1528 records. After screening based on titles and abstracts, eight articles remained for full-text assessment (Additional file 1: Figure S2). All of them met the inclusion criteria and were included in the systematic review [4, 9-15]. Details of the key characteristics of the trials are presented in Additional file 1: Table S1. Seven RCTs included more than 100 patients $[4,9,12,15]$ and one trial was multicenter [9]. The clinical settings were cardiac surgery in four trials $[10,11,13,15]$, general surgery in three studies $[4,9,12]$ and hepatic surgery in one trial. The duration of the GDHT varied from 4 to $12 \mathrm{~h}$. Fluids and inotropic support were used within the GDHT protocol in all included studies, while some of the RCTs also used RBC transfusion and vasodilators. Most included trials were categorized as at low risk of bias (Additional file 1: Table S2 and Figure S3). 
Table 1 Baseline and demographic patients' characteristics

\begin{tabular}{|c|c|c|}
\hline Variable & $\begin{array}{l}\text { GDHT group } \\
(n=64)\end{array}$ & $\begin{array}{l}\text { Usual care } \\
\text { group }(n=64)\end{array}$ \\
\hline Age (years), mean $\pm S D$ & $66 \pm 12$ & $68 \pm 11$ \\
\hline BMI $\left(\mathrm{kg} / \mathrm{m}^{2}\right)$, median (IQR) & $25(21-31)$ & $25(21-30)$ \\
\hline Sex (male), $n(\%)$ & $32(50 \%)$ & 37 (58\%) \\
\hline Race (white), n (\%) & $48(75 \%)$ & $52(81 \%)$ \\
\hline $\begin{array}{l}\text { Charlson score } \\
\text { median (IQR) }\end{array}$ & $5(4-6)$ & $6(5-8)$ \\
\hline \multicolumn{3}{|l|}{ ECOG score ${ }^{\mathrm{b}}, n(\%)$} \\
\hline 0 & $29(47 \%)$ & $26(43 \%)$ \\
\hline 1 & 19 (31\%) & $19(32 \%)$ \\
\hline 2 & $6(10 \%)$ & $8(13 \%)$ \\
\hline 3 & $8(13 \%)$ & $6(10 \%)$ \\
\hline 4 & 0 & $1(2 \%)$ \\
\hline $\begin{array}{l}\text { Karnofsky scorec, } \\
\text { median (IQR) }\end{array}$ & $90(80-100)$ & $90(80-100)$ \\
\hline \multicolumn{3}{|l|}{ Neoplasm, n (\%) } \\
\hline Stomach & 0 & $1(2 \%)$ \\
\hline Esophagus & $9(13 \%)$ & $6(10 \%)$ \\
\hline Colon-rectus & $8(13 \%)$ & $6(10 \%)$ \\
\hline Gynecological & $18(30 \%)$ & $21(34 \%)$ \\
\hline Pancreas & $9(15 \%)$ & $9(15 \%)$ \\
\hline Liver & $6(10 \%)$ & $2(3 \%)$ \\
\hline Prostate & $6(10 \%)$ & $8(13 \%)$ \\
\hline Kidney & $4(7 \%)$ & $5(8 \%)$ \\
\hline Others & $1(2 \%)$ & $3(5 \%)$ \\
\hline $\begin{array}{l}\text { Recent chemotherapy, } \\
n(\%)\end{array}$ & $3(5 \%)$ & $1(2 \%)$ \\
\hline $\begin{array}{l}\text { Recent radiotherapy, } \\
n(\%)\end{array}$ & 0 & 0 \\
\hline \multicolumn{3}{|l|}{ Smokers, $n(\%)$} \\
\hline No & $41(64 \%)$ & $41(64 \%)$ \\
\hline Yes & $9(14 \%)$ & $7(11 \%)$ \\
\hline Previously & $14(22 \%)$ & $16(25 \%)$ \\
\hline \multicolumn{3}{|l|}{ Alcoholism, n (\%) } \\
\hline No & $56(88 \%)$ & $55(86 \%)$ \\
\hline Yes & $4(6 \%)$ & $2(33 \%)$ \\
\hline Previously & $4(6 \%)$ & $7(11 \%)$ \\
\hline Hypertension, n (\%) & 38 (59\%) & $35(55 \%)$ \\
\hline Diabetes mellitus, $n$ (\%) & $12(19 \%)$ & $16(25 \%)$ \\
\hline Heart failure, $n(\%)$ & $2(3 \%)$ & $3(5 \%)$ \\
\hline $\begin{array}{l}\text { Chronic obstructive } \\
\text { pulmonary disease, } n(\%)\end{array}$ & $4(6 \%)$ & $6(9 \%)$ \\
\hline $\begin{array}{l}\text { Chronic kidney disease, } \\
n(\%)\end{array}$ & $2(3 \%)$ & $6(9 \%)$ \\
\hline Hypothyroidism, n (\%) & $5(8 \%)$ & $4(6 \%)$ \\
\hline Stroke, $n(\%)$ & 0 & $6(9 \%)$ \\
\hline
\end{tabular}

Table 1 Baseline and demographic patients' characteristics (Continued)

\begin{tabular}{|c|c|c|}
\hline Variable & $\begin{array}{l}\text { GDHT group } \\
(n=64)\end{array}$ & $\begin{array}{l}\text { Usual care } \\
\text { group }(n=64)\end{array}$ \\
\hline $\begin{array}{l}\text { Acute myocardial } \\
\text { infarction, } n(\%)\end{array}$ & $4(6 \%)$ & $5(8 \%)$ \\
\hline $\begin{array}{l}\text { Pulmonary embolism/ } \\
\text { deep vein thrombosis, } \\
n(\%)\end{array}$ & 0 & $1(2 \%)$ \\
\hline \multicolumn{3}{|c|}{ 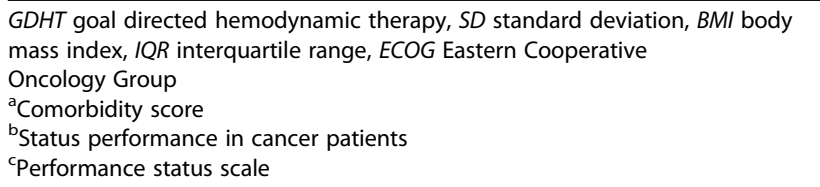 } \\
\hline
\end{tabular}

There were no differences in the longest follow-up mortality between groups (RR $0.85,95 \%$ CI $0.59-1.23 ; p$ for effect $=0.4 ; p$ for heterogeneity $=0.7 ; I^{2}=0 \%$; eight included trials; Fig. 3a) and overall complication rate (RR $0.88,95 \%$ CI $0.71-1.08 ; p$ for effect $=0.2$; $p$ for heterogeneity $=0.07 ; I^{2}=48 \%$; seven included trials; Fig. 3b). The lack of a GDHT effect on the overall complication rate was confirmed when sequentially removing included trials (Additional file 1: Table S3). GDHT reduced hospital LOS (MD - 1.6; 95\% CI 2.75 to $-0.46 ; p$ for effect $=0.006$; $p$ for heterogeneity $=0.002 ; I^{2}=74 \%$; six included trials; Fig. $3 \mathrm{c}$ ).

\section{Discussion}

The main finding of this RCT is that among cancer patients undergoing high-risk surgery, the use of CI-guided

Table 2 Surgical-related characteristics

\begin{tabular}{|c|c|c|}
\hline Variable & $\begin{array}{l}\text { GDHT group } \\
(n=64)\end{array}$ & $\begin{array}{l}\text { Usual care group } \\
(n=64)\end{array}$ \\
\hline \multicolumn{3}{|l|}{ Surgery type, n (\%) } \\
\hline Upper gastrointestinal tract & $21(33 \%)$ & $13(20 \%)$ \\
\hline Lower gastrointestinal tract & $15(23 \%)$ & $14(22 \%)$ \\
\hline Liver and biliary tract & $2(3 \%)$ & $5(8 \%)$ \\
\hline Urogenital tract & $15(23 \%)$ & $22(34 \%)$ \\
\hline Others & $11(17 \%)$ & $10(16 \%)$ \\
\hline $\begin{array}{l}\text { Duration of surgery } \\
\text { (min), median (IQR) }\end{array}$ & $360(273-480)$ & $300(240-435)$ \\
\hline Fluids (ml), median (IQR) & $4000(3000-5763)$ & $4000(3000-5225)$ \\
\hline Norepinephrine, $n$ (\%) & $20(31 \%)$ & $19(30 \%)$ \\
\hline Dobutamine, $n$ (\%) & 0 & $4(6 \%)$ \\
\hline Vasopressin, $n(\%)$ & $1(2 \%)$ & 0 \\
\hline RBC transfusion, $n(\%)$ & $19(30 \%)$ & $15(23 \%)$ \\
\hline Fresh frozen plasma, $n(\%)$ & $2(3 \%)$ & $2(3 \%)$ \\
\hline Cryoprecipitate, $n$ (\%) & 0 & $1(2 \%)$ \\
\hline Platelets, $n$ (\%) & $1(2 \%)$ & 0 \\
\hline
\end{tabular}

$G D H T$ goal directed hemodynamic therapy, $I Q R$ interquartile range, $R B C$ red blood cell 
Table 3 Hemodynamic variables and tissue perfusion markers during 8-h intervention period

\begin{tabular}{|c|c|c|c|}
\hline Variable & $\begin{array}{l}\text { GDHT group } \\
(n=64)\end{array}$ & $\begin{array}{l}\text { Usual care group } \\
(n=64)\end{array}$ & $p$ \\
\hline \multicolumn{3}{|l|}{$\Delta \mathrm{PCO}_{2}(\%)$} & 0.9 \\
\hline $\mathrm{Oh}$ & $6.8 \pm 4.4$ & $6.8 \pm 4.9$ & \\
\hline $2 \mathrm{~h}$ & $7.5 \pm 5.8$ & $6.9 \pm 4.7$ & \\
\hline $8 \mathrm{~h}$ & $6.0 \pm 3.3$ & $6.8 \pm 4.2$ & \\
\hline \multicolumn{3}{|l|}{ BE (mmol) } & 0.9 \\
\hline $\mathrm{Oh}$ & $-3.7 \pm 2.9$ & $-3.7 \pm 3.0$ & \\
\hline $2 \mathrm{~h}$ & $-4.1 \pm 3.7$ & $-3.4 \pm 2.9$ & \\
\hline $8 \mathrm{~h}$ & $-2.4 \pm 2.7$ & $-2.9 \pm 3.0$ & \\
\hline \multicolumn{3}{|c|}{ Lactate (mmol/L) } & 0.9 \\
\hline $\mathrm{Oh}$ & $2.6 \pm 1.5$ & $2.8 \pm 2.3$ & \\
\hline $2 \mathrm{~h}$ & $2.9 \pm 2.0$ & $3.0 \pm 2.3$ & \\
\hline $8 \mathrm{~h}$ & $2.9 \pm 1.8$ & $3.0 \pm 2.5$ & \\
\hline \multicolumn{3}{|l|}{ HR (bpm) } & 0.9 \\
\hline $\mathrm{Oh}$ & $83.1 \pm 21.6$ & $85.5 \pm 23.9$ & \\
\hline $2 \mathrm{~h}$ & $82.9 \pm 20.7$ & $83.9 \pm 22.6$ & \\
\hline $8 \mathrm{~h}$ & $88.2 \pm 21.3$ & $82.9 \pm 18.9$ & \\
\hline \multicolumn{3}{|c|}{ MAP (mmHg) } & 0.3 \\
\hline $\mathrm{Oh}$ & $94.5 \pm 19.6$ & $91.6 \pm 17.8$ & \\
\hline $2 \mathrm{~h}$ & $88.1 \pm 15.6$ & $87.3 \pm 14.7$ & \\
\hline $8 \mathrm{~h}$ & $85.3 \pm 16.9$ & $82.1 \pm 15.2$ & \\
\hline \multicolumn{3}{|l|}{$\mathrm{ScvO}_{2}(\%)$} & 0.2 \\
\hline $\mathrm{Oh}$ & $75.7 \pm 9.0$ & $77.1 \pm 9.0$ & \\
\hline $2 \mathrm{~h}$ & $75.6 \pm 8.7$ & $77.4 \pm 6.6$ & \\
\hline $8 \mathrm{~h}$ & $78.7 \pm 6.6$ & $76.4 \pm 8.0$ & \\
\hline \multicolumn{3}{|c|}{$\mathrm{Cl}\left(\mathrm{L} / \mathrm{min} / \mathrm{m}^{2}\right)$} & \\
\hline $\mathrm{Oh}$ & $2.9 \pm 1.0$ & - & \\
\hline $2 \mathrm{~h}$ & $2.9 \pm 0.9$ & - & \\
\hline $8 \mathrm{~h}$ & $3.1 \pm 1.0$ & - & \\
\hline
\end{tabular}

Data expressed as mean \pm standard deviation

$G D H T$ goal-directed hemodynamic therapy, $\triangle P \mathrm{PO}_{2}$ dioxide carbon gap, $B E$ base excess, $\mathrm{HR}$ heart rate, MAP mean arterial pressure, $\mathrm{ScvO}_{2}$ central venous oxygen saturation, $\mathrm{Cl}$ cardiac index

hemodynamic therapy applied in the first 8 postoperative hours is not associated with a reduction in 30-day mortality and severe postoperative complications when compared with protocolized hemodynamic therapy incorporating measures of perfusion and intraoperative PPV. The GDHT resulted in a higher exposure of the patients to dobutamine, without improving outcomes. After including the results of this trial in an updated systematic review and meta-analysis, we confirmed that the strategy of postoperative GDHT does not reduce overall complications and mortality in high-risk surgical patients. However, the meta-analysis suggested that postoperative GHDT is associated with a lower length of hospital stay.
GDHT is recommended in the context of enhanced recovery programs in high-risk patients [16]. A recent published meta-analysis including $45 \mathrm{RCTs}$ suggested that the use of GDHT in major abdominal surgery reduces mortality and overall complications [6]. Our meta-analysis differs from this previous one because we included RCTs with a GDTH protocol performed exclusively after surgery, in the ICU. Most studies included in the meta-analysis by Sun et al. [6] start the protocol during surgery and extend it to the first $8 \mathrm{~h}$ of the postoperative period.

Interestingly, the best timing for performing GDHT is not specified in previous systematic reviews and recommendations. The majority of studies defined the interventions as "perioperative", including the surgical room period and the first 6-12 h of postoperative care. We therefore speculate that most of the benefits of the GDHT protocol, if any, are the result of intraoperative protocols. Our study demonstrates that a GDHT postoperative protocol does not add benefits in outcomes when compared to a usual care protocol and might be associated with over therapy without improving outcomes.

The updated meta-analysis including nine randomized studies confirmed no reduction in mortality and overall complications while suggesting that the benefits of postoperative GDHT might be limited to a reduction in the length of hospital stay. This brings to the discussion whether it is necessary to apply postoperative GDHT in high-risk patients already undergoing an intraoperative protocol of cardiac output-guided hemodynamic algorithm as we did in our patients.

Our study differs from previous ones performed in noncardiac surgery [3-5, 7]. First, the usual care group received multimodal hemodynamic care based on the optimization of hemodynamic parameters and tissue perfusion markers, which may be enough for adequate oxygen delivery during the postoperative period. Second, during anesthesia, patients were monitored with a central venous line and an arterial line, and hemodynamic variables such as $\mathrm{PPV}, \mathrm{ScvO}_{2}$ and arterial lactate were evaluated in protocolized care. Thus, it is possible that occult tissue hypoperfusion and hypoxia were avoided in the subsequent $8 \mathrm{~h}$ of the intervention. This is confirmed by the observation that the majority of patients were admitted to the ICU with $\mathrm{CI}>2.5 \mathrm{~L} / \mathrm{min} / \mathrm{m}^{2}$.

In septic shock patients, Rivers et al. [17] demonstrated that goal-directed therapy aiming at adequate $\mathrm{ScvO}_{2}$ reduced mortality. In the last years, with advances in the process of care, more recent studies have not confirmed the benefits of applying GDHT in the ICU in septic patients [18].

The largest study evaluating the impact of cardiac output-guided hemodynamic therapy in surgical 
Table 4 Primary outcome

\begin{tabular}{|c|c|c|c|c|}
\hline Variable & $\begin{array}{l}\text { GDHT group } \\
(n=64)\end{array}$ & $\begin{array}{l}\text { Usual care group } \\
(n=64)\end{array}$ & $\begin{array}{l}\text { Absolute } \\
\text { difference }(95 \% \mathrm{Cl})\end{array}$ & $p$ \\
\hline Composite endpoint & $53(40-66)$ & $44(31-57)$ & $9.4(-7.8$ to 25.8$)$ & 0.3 \\
\hline Mortality & $14(7-25)$ & $9(4-19)$ & $4.7(-6.9$ to 16.4$)$ & 0.4 \\
\hline Stroke & $0(0-6)$ & $2(0-8)$ & $-1.6(-8.3$ to 4.2$)$ & 0.9 \\
\hline AKI & $51(36-61)$ & $39(27-52)$ & $9.4(-7.6$ to 25.6$)$ & 0.2 \\
\hline ARDS & $5(2-15)$ & $2(0-8)$ & $4.7(-3.1$ to 13.5$)$ & 0.4 \\
\hline Acute myocardial infarction & $2(0-8)$ & $5(0.9-13)$ & $-3.1(-11.4$ to 4.3$)$ & 0.6 \\
\hline Acute heart failure & $0(0-6)$ & $2(0-8)$ & $-1.6(-8.3$ to 4.2$)$ & 0.5 \\
\hline Mesenteric ischemia & $2(0-8)$ & $0(0-6)$ & $1.6(-4.2$ to 8.3$)$ & 0.9 \\
\hline Peripheral vascular ischemia & $0(0-6)$ & $3(0.3-11)$ & $-3.1(-10.7$ to 3.0$)$ & 0.5 \\
\hline Pulmonary embolism & $0(0-6)$ & $0(0-6)$ & - & - \\
\hline Deep wound infection & $3(0.3-11)$ & $8(3-17)$ & $-4.7(-14.2$ to 4.1$)$ & 0.4 \\
\hline Reoperation & $8(3-17)$ & $5(0.9-13)$ & $3.1(-6.2$ to 12.8$)$ & 0.7 \\
\hline
\end{tabular}

Data presented as \% (95\% Cl)

GDT goal-directed hemodynamic therapy, $C l$ confidence interval, $A K I$ acute kidney injury, ARDS acute respiratory distress syndrome

patients, the OPTIMISE trial, included 734 patients undergoing major abdominal surgery in 17 different centers. This study did not show any benefits in the intervention group for postoperative complications and mortality reduction over standard therapy [7].

In our GDHT group, patients needed more dobutamine, but we did not observe a higher risk of arrhythmia or myocardial injury, as confirmed by previous trials $[7,19]$. Our GDHT protocol of care did not result in either more prescribed fluids or red blood cells, maybe because most patients were already adequately resuscitated during the intraoperative period.

We used albumin solution in the algorithm of treatment because colloid solutions are superior when compared to crystalloids in reaching hemodynamic goals
[20]. In addition, previous randomized clinical studies in critically ill patients showed the safety of albumin solutions [21, 22].

We chose to use a minimally invasive noncalibrated device to measure the cardiac index. Although this device has its accuracy reduced when compared to other calibrated devices, the data are reproducible, easy to measure and easy to interpret and it is used in both surgical rooms and ICUs. In addition, physicians and nurses were already trained. We selected a cardiac index target of $2.5 \mathrm{~L} / \mathrm{min} / \mathrm{m}^{2}$ based on a previous study that was able to demonstrate improved outcomes in high-risk patients using a similar target [12].

Our study has limitations. Due to the nature of the intervention protocol, blinding was not feasible, and to

Table 5 Secondary outcomes

\begin{tabular}{|c|c|c|c|c|}
\hline Variable & $\begin{array}{l}\text { GDHT group } \\
(n=64)\end{array}$ & $\begin{array}{l}\text { Usual care group } \\
(n=64)\end{array}$ & Absolute difference $(95 \% \mathrm{Cl})$ & $p$ \\
\hline Septic shock & $16(8-27)$ & $13(6-23)$ & $3.1(-9.3$ to 15.5$)$ & 0.611 \\
\hline AKI AKIN 3 and RRT & $13(6-23)$ & $8(3-17)$ & $4.7(-6.3$ to 15.9$)$ & 0.380 \\
\hline ICU readmission & $13(6-23)$ & $9(4-19)$ & $3.1(-8.2$ to 14.6$)$ & 0.571 \\
\hline $\begin{array}{l}\text { Length of ICU stay (days), } \\
\text { median (IQR) }\end{array}$ & $3(2-8)$ & $3(2-5)$ & - & 0.571 \\
\hline $\begin{array}{l}\text { Length of hospital stay (days), } \\
\text { median (IQR) }\end{array}$ & $11(6-19)$ & $10(6-15)$ & - & 0.354 \\
\hline SOFA score, mean \pm SD & & & & 0.308 \\
\hline Admission & $4.3 \pm 2.4$ & $3.6 \pm 2.2$ & $-0.7(-1.5$ to 0.1$)$ & \\
\hline D1 & $4.0 \pm 2.5$ & $3.2 \pm 2.3$ & $-0.8(-1.6$ to 0$)$ & \\
\hline D2 & $2.8 \pm 2.8$ & $2.1 \pm 2.2$ & $-0.7(-1.6$ to 0.2$)$ & \\
\hline
\end{tabular}

Data presented as \% (95\% Cl)

GDT goal-directed hemodynamic therapy, $C I$ confidence interval, AKI acute kidney injury, AKIN Acute Kidney Injury Network, RRT renal replacement therapy, ICU intensive care unit, IQR interquartile range, SOFA Sequential Organ Failure Assessment, SD standard deviation, D1 first day postoperative,

D2 second day postoperative 

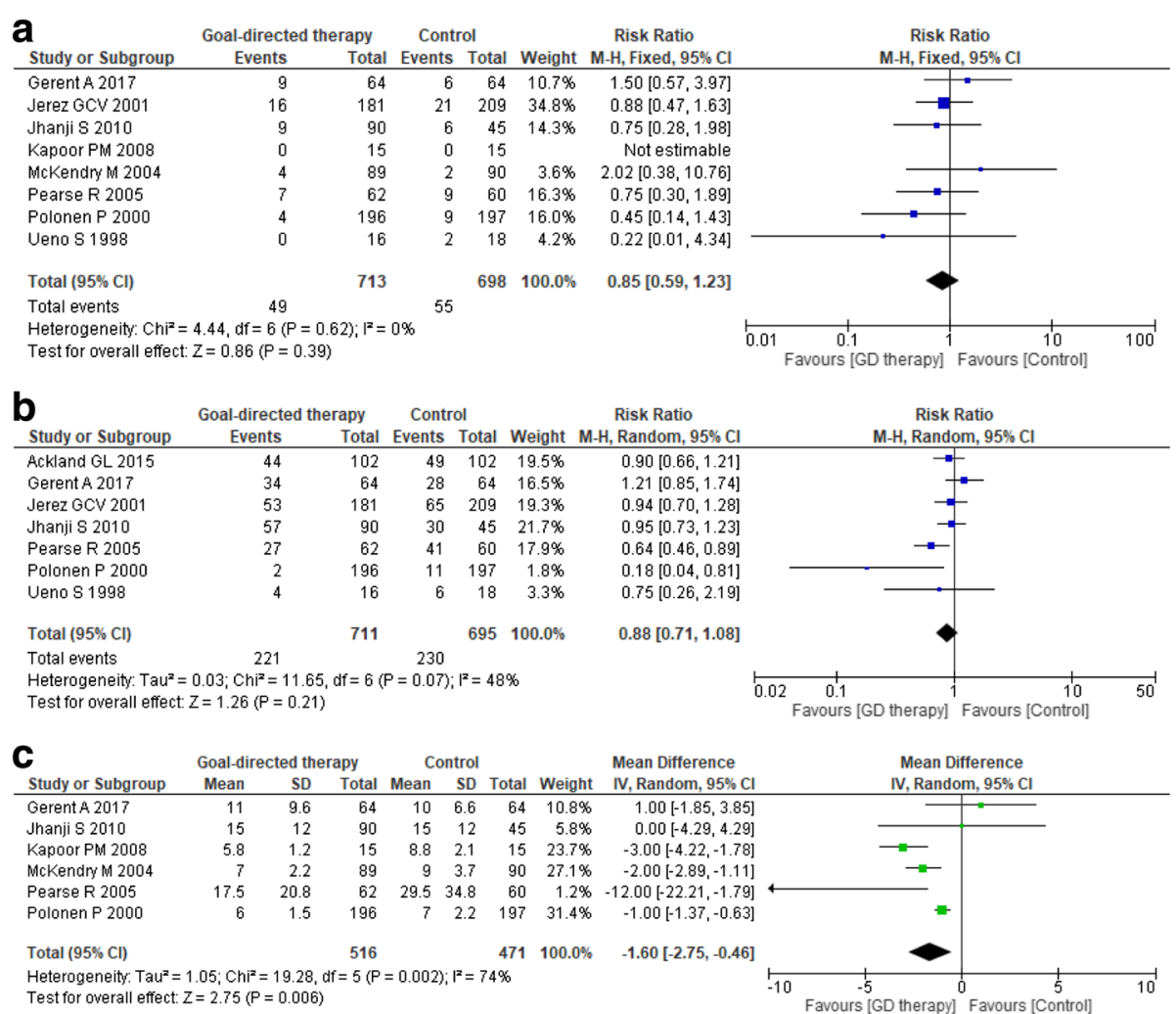

Fig. 3 Forest plot of a longest follow-up mortality, $\mathbf{b}$ overall complication rate and $\mathbf{c}$ hospital length of stay. M-H Mantel-Haenszel method, Cl confidence interval, GD goal-directed, IV inverse variance method

reduce potential bias, outcome assessors were not aware of the study group assignment. Our results may have limited external validity because this was a single-center trial conducted in a cancer reference hospital. In addition, we analyzed a conventional hemodynamic perioperative protocol based on "normal" values of variables such as cardiac index (CI) and systolic volume. However, we have learned that population-based "normal" values do not necessarily represent the optimal values or the personal normal values of an individual. It is known that many hemodynamic variables have marked interindividual variability and depend on other factors such as biometric factors and time of disease, and probably using fixed hemodynamic goals might have contributed to the negative results of this trial. We postulate that the best concept to be applied at the bedside in high-surgical risk patients is personalized hemodynamic management, based on adaptive multiparameter hemodynamic optimization strategies targeting individual normal values adapted to the clinical situation [23-25].

This was a pragmatic study designed to analyze the effect of GDHT in the postoperative scenario of the ICU. We aimed to assess the postoperative period separately because in usual practice, independently of intraoperative management, the hemodynamic algorithm continues to be applied routinely during the first hours in the ICU. We evaluated whether applying a fixed GDHT algorithm in the postoperative period might contribute to reduce complications or even results in harm. Our results bring into discussion the need for a more personalized approach at this point, once we have shown that there is no benefit of postoperative GDHT in cancer patients undergoing major surgeries. The updated meta-analysis confirms that postoperative GDHT does not reduce overall complications and death, but might be associated with a reduced length of hospital stay.

\section{Conclusions}

A cardiac index-guided hemodynamic therapy applied in the first 8 postoperative hours in cancer patients undergoing high-risk surgery did not reduce 30-day mortality and the rate of postoperative complications when compared with protocolized hemodynamic therapy including measures of perfusion and intraoperative PPV. This therapy was associated with an increased use of dobutamine without improving outcomes. 


\section{Additional file}

Additional file 1: Effect of postoperative goal-directed therapy in cancer patients undergoing high-risk surgery: randomized clinical trial and meta-analysis. Appendix 1. Surgical, anesthetic technique and intensive care treatment. Appendix 2. Outcome definitions. Appendix 3. Data collection. Appendix 4. Systematic review details. Figure S1. Cardiac index during intervention in the GDHT group (median and interquartile range). Figure S2. Study flow diagram of systematic review. Figure S3. Risk of bias graph: review authors' judgments about each risk of bias item presented as percentages across all included studies. Table S1. Characteristics of included trials. Table S2. Risk of bias. Table S3. Sensitivity analysis for overall complication rate by sequential removal of each trial. Supplemental references. (DOC 255 kb)

\section{Abbreviations}

AKl: Acute kidney injury; ARDS: Acute respiratory distress syndrome; $\mathrm{Cl}$ : Cardiac index; $\mathrm{DO}_{2}$ : Oxygen delivery; GDHT: Goal-directed hemodynamic therapy; HR: Heart rate; ICU: Intensive care unit; IQR: Interquartile range; MAP: Mean arterial pressure; RBC: Red blood cell; RCT: Randomized clinical trial; RR: Risk ratio; RRT: Renal replacement therapy; $\mathrm{ScvO}_{2}$ : Central venous oxygen saturation; SD: Standard deviation; SOFA: Sepsis-related organ failure assessment; SVI: Stroke volume index

\section{Funding}

The two funding sources were the University of Sao Paulo (Brazil) and Edwards LTDA (Irvine, CA, USA), which had no other role in the study.

\section{Availability of data and materials}

All data generated or analyzed during this study are included in this published article and its supplementary information files.

\begin{abstract}
Authors' contributions
ARMG had full access to all of the data and takes responsibility for the integrity of the data and the accuracy of the data analysis. ARMG, JPA, FRBGG and LAH contributed to study concept and design. All authors contributed to acquisition, analysis or interpretation of data. EPMdA, ARMG, JPA, FRBGG, LAH, EF and GL drafted the manuscript. EPMdA, ARMG, JPA, FRBGG, LAH, EF, GL and URJ contributed to critical revision of the manuscript for important intellectual content. JTF and EF performed statistical analysis. ARMG, EF, CMS and CLP contributed administrative, technical or material support. ARMG and LAH were responsible for study supervision. All authors gave final approval of the version to be published. All authors agreed to be accountable for all aspects of the work, thereby ensuring that questions related to the accuracy or integrity of any part of the work are appropriately investigated and resolved.
\end{abstract}

\section{Ethics approval and consent to participate}

The study was approved by the Faculty of Medicine Ethics Committee (number 335/13). Written informed consent was obtained from all subjects or their legal surrogates prior to enrolment in the study.

\section{Competing interests}

The authors declare that they have no competing interests.

\section{Publisher's Note}

Springer Nature remains neutral with regard to jurisdictional claims in published maps and institutional affiliations.

\section{Author details}

${ }^{1}$ Intensive Care Unit and Department of Anesthesiology, Instituto do Cancer, Hospital das Clinicas da Faculdade de Medicina da Universidade de Sao Paulo, Sao Paulo, Brazil. ²Department of Anesthesia and Intensive Care, IRCCS San Raffaele Scientific Institute, Milan, Italy. ${ }^{3}$ Department of Surgery, Instituto do Cancer, Hospital das Clinicas da Faculdade de Medicina da Universidade de Sao Paulo, Sao Paulo, Brazil. ${ }^{4}$ Department of Cardiopneumology, Instituto do Coracao, Hospital das Clinicas, Faculdade de Medicina da Universidade de Sao Paulo, Sao Paulo, Brazil.
Received: 17 November 2017 Accepted: 2 May 2018

Published online: 23 May 2018

\section{References}

1. de Almeida JP, Vincent JL, Galas FR, et al. Transfusion requirements in surgical oncology patients: a prospective, randomized controlled trial. Anesthesiology. 2015;122:29-38.

2. Sankar A, Beattie WS, Wijeysundera DN. How can we identify the high-risk patient? Curr Opin Crit Care. 2015;21:328-35.

3. Tote SP, Grounds RM. Performing perioperative optimization of the high-risk surgical patient. Br J Anaesth. 2006;97:4-11.

4. Jhanji S, Vivian-Smith A, Lucena-Amaro S, Watson D, Hinds CJ, Pearse RM. Haemodynamic optimisation improves tissue microvascular flow and oxygenation after major surgery: a randomised controlled trial. Crit Care. 2010;14:R151.

5. Benes J, Chytra I, Altmann P, et al. Intraoperative fluid optimization using stroke volume variation in high risk surgical patients: results of prospective randomized study. Crit Care. 2010;14:R118.

6. Sun Y, Chai F, Pan C, Romeiser JL, Gan TJ. Effect of perioperative goaldirected hemodynamic therapy on postoperative recovery following major abdominal surgery - a systematic review and meta-analysis of randomized controlled trials. Crit Care. 2017:21:141.

7. Pearse RM, Harrison DA, MacDonald N, et al. Effect of a perioperative, cardiac output-guided hemodynamic therapy algorithm on outcomes following major gastrointestinal surgery: a randomized clinical trial and systematic review. JAMA. 2014;311:2181-90.

8. Grocott MP, Dushianthan A, Hamilton MA, et al. Perioperative increase in global blood flow to explicit defined goals and outcomes following surgery. Cochrane Database Syst Rev. 2012;11:CD004082.

9. Ackland $G L$, lqbal $S$, Paredes $L G$, et al. Individualised oxygen delivery targeted haemodynamic therapy in high-risk surgical patients: a multicentre, randomised, double-blind, controlled, mechanistic trial. Lancet Respir Med. 2015:3:33-41.

10. Gómez-Coronado VJ, Marcos MR, Civantos DP, et al. Hemodynamic optimazation and morbimortality after heart surgery. Med Int. 2001;25:297-302.

11. Kapoor PM, Kakani M, Chowdhury U, Choudhury M, Lakshmy, Kiran U. Early goal-directed therapy in moderate to high-risk cardiac surgery patients. Ann Card Anaesth. 2008;11:27-34.

12. Pearse R, Dawson D, Fawcett J, Rhodes A, Grounds RM, Bennett ED. Early goal-directed therapy after major surgery reduces complications and duration of hospital stay. A randomised, controlled trial [ISRCTN38797445]. Crit Care. 2005;9:R687-93.

13. Polonen $\mathrm{P}$, Ruokonen $\mathrm{E}$, Hippelainen $\mathrm{M}$, Poyhonen M, Takala J. A prospective, randomized study of goal-oriented hemodynamic therapy in cardiac surgical patients. Anesth Analg. 2000;90:1052-9.

14. Ueno S, Tanabe G, Yamada H, et al. Response of patients with cirrhosis who have undergone partial hepatectomy to treatment aimed at achieving supranormal oxygen delivery and consumption. Surgery. 1998;123:278-86.

15. McKendry M, McGloin H, Saberi D, Caudwell L, Brady AR, Singer M. Randomised controlled trial assessing the impact of a nurse delivered, flow monitored protocol for optimisation of circulatory status after cardiac surgery. BMJ. 2004;329:258.

16. Hamilton MA, Cecconi M, Rhodes A. A systematic review and meta-analysis on the use of preemptive hemodynamic intervention to improve postoperative outcomes in moderate and high-risk surgical patients. Anesth Analg. 2011;112:1392-402.

17. Rivers E, Nguyen B, Havstad S, et al. Early goal-directed therapy in the treatment of severe sepsis and septic shock. N Engl J Med. 2001;345:1368-77.

18. PRISM Investigators, Rowan KM, Angus DC, et al. Early, goal-directed therapy for septic shock - a patient-level meta-analysis. N Engl J Med. 2017;376: 2223-34.

19. Osawa EA, Rhodes A, Landoni G, et al. Effect of perioperative goal-directed hemodynamic resuscitation therapy on outcomes following cardiac surgery: a randomized clinical trial and systematic review. Crit Care Med. 2016;44: 724-33.

20. Feldheiser A, Pavlova V, Bonomo T, et al. Balanced crystalloid compared with balanced colloid solution using a goal-directed haemodynamic algorithm. Br J Anaesth. 2013;110:231-40.

21. Finfer $\mathrm{S}$, Bellomo R, Boyce $\mathrm{N}$, et al. A comparison of albumin and saline for fluid resuscitation in the intensive care unit. N Engl J Med. 2004;350:2247-56. 
22. Caironi P, Tognoni G, Masson S, et al. Albumin replacement in patients with severe sepsis or septic shock. N Engl J Med. 2014;370:1412-21.

23. Saugel B, Vincent JL, Wagner JY. Personalized hemodynamic management. Curr Opin Crit Care. 2017;23:334-41.

24. Molnar Z, Szabo Z, Nemeth M. Multimodal individualized concept of hemodynamic monitoring. Curr Opin Anaesthesiol. 2017;30:171-7.

25. Ince C. Personalized physiological medicine. Crit Care. 2017;21:308.

- fast, convenient online submission

- thorough peer review by experienced researchers in your field

- rapid publication on acceptance

- support for research data, including large and complex data types

- gold Open Access which fosters wider collaboration and increased citations

- maximum visibility for your research: over $100 \mathrm{M}$ website views per year 